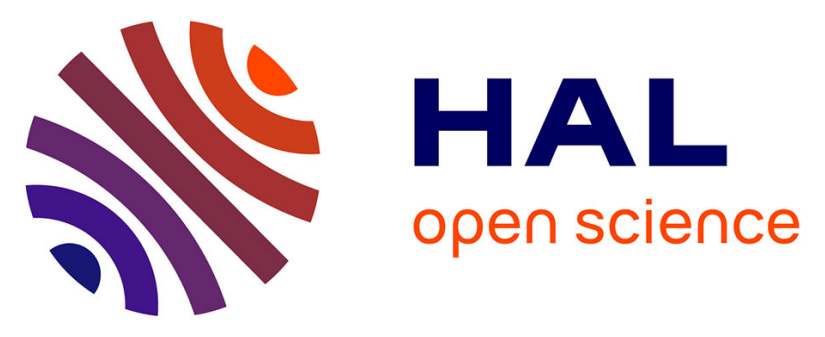

\title{
Mapping of the electrostatic potentials in MOCVD and hybrid GaN tunnel junctions for InGaN/GaN blue emitting light emitting diodes by off-axis electron holography correlated with structural, chemical, and optoelectronic characterization
}

D. Cooper, V. Fan Arcara, B. Damilano, L. Amichi, A. Mavel, N. Rochat, G. Feuillet, A. Courville, S. Vézian, V Fan Arcara, et al.

\section{- To cite this version:}

D. Cooper, V. Fan Arcara, B. Damilano, L. Amichi, A. Mavel, et al.. Mapping of the electrostatic potentials in MOCVD and hybrid GaN tunnel junctions for InGaN/GaN blue emitting light emitting diodes by off-axis electron holography correlated with structural, chemical, and optoelectronic characterization. Journal of Applied Physics, 2021, 130 (2), pp.025704. 10.1063/5.0054810 . hal-03418879

\author{
HAL Id: hal-03418879 \\ https://hal.science/hal-03418879
}

Submitted on 8 Nov 2021

HAL is a multi-disciplinary open access archive for the deposit and dissemination of scientific research documents, whether they are published or not. The documents may come from teaching and research institutions in France or abroad, or from public or private research centers.
L'archive ouverte pluridisciplinaire HAL, est destinée au dépôt et à la diffusion de documents scientifiques de niveau recherche, publiés ou non, émanant des établissements d'enseignement et de recherche français ou étrangers, des laboratoires publics ou privés. 
Mapping of the electrostatic potentials in MOCVD and hybrid GaN tunnel junctions for InGaN/GaN

blue emitting light emitting diodes by off-axis electron holography correlated with structural, chemical and optoelectronic characterization.

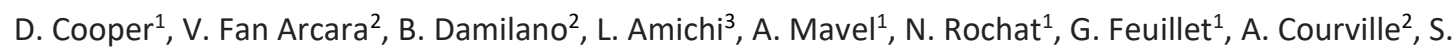
Vézian ${ }^{2}$ and J.Y. Duboz ${ }^{2}$

${ }^{1}$ Univ. Grenoble Alpes, CEA, LETI, F-38000 Grenoble, France.

${ }^{2}$ Université Côte D’Azur, CNRS, CRHEA, Rue B. Gregory, Valbonne, France.

${ }^{3}$ Univ. Grenoble Alpes, CEA, INAC, F-38000 Grenoble, France.

Corresponding author david.cooper@cea.fr

Off-axis electron holography has been used to measure the width of the depletion region in a series of Tunnel Junction GaN light emitting diodes that have been prepared using different growth processes for blue emission. The total measured potentials are combinations of the mean inner potential, dopant potential and piezoelectric contributions. The dopant potential has been unmixed from the mean inner potential such that the width of the tunnel Junctions in the different diodes can be measured. The experimental results are then compared to secondary ion mass spectrometry, simulations and optoelectronic testing. We find that the measured tunnel junction widths are consistent with simulations as well as the current density and voltage characteristics. As such, off-axis electron holography has been demonstrated as a unique technique that can be used to reproducibly measure the electrostatic potentials in tunnel junctions with $\mathrm{nm}$-scale resolution in real III-V device specimens.

\section{Introduction}

The implementation of tunnel junctions (TJs) can be used to solve several issues that affect the design of efficient GaN-based optoelectronic devices. In particular, for the replacement of the p-type contact layers by $n$-type layers which improve the current uniformity due to their better conductivity. ${ }^{1,2}$ The substitution of the highly resistive ohmic $\mathrm{p}$-contact by a low resistivity $\mathrm{n}$-contact will also reduce the total resistivity of the diode. ${ }^{3}$ They can be used to replace the highly absorbing p-GaN contact layer in deep-UV light emitting diodes such that a n-AIGaN contact layer can improve the extraction efficiency of these devices. ${ }^{4-6}$ TJs can be also used to connect different active regions to produce tandem and monolithic white LEDs ${ }^{7,8}$ or to connect different active regions through the realization of intra cavity $n$-contacts in vertical-cavity surface emitting lasers (VCSELs) ${ }^{9}$. All these applications, require a low resistivity tunnel junction to obtain efficient devices. Therefore, it is necessary to produce TJs with narrow depletion widths since the tunneling probability exponentially decreases with the width of the junction. ${ }^{10,11}$

To form narrow depletion regions in the TJs, high active dopant concentrations are required. The use of interlayers to induce the formation of polarization sheet charges at the interface have also been proven to significantly enhance the tunnel behavior. ${ }^{12,13}$ Generally, the improvement of the tunneling behavior 
by these methods is demonstrated by the superior quality of the electro-optical characteristics of the TJLEDs in comparison to their counterparts. However, it is complicated to measure the opto-electrical properties of the LEDs and directly link them to the electrical activity of the dopants and width of the depletion region. Techniques such as SIMS and atom probe tomography ${ }^{14}$ can provide measurements of the total dopant concentration and the distribution of the dopants. However, they do not provide a direct measurement of their electrical activity. In particular, the electrical activity of $\mathrm{Mg}$ in nitrides remains uncertain as it can be reduced by passivation by hydrogen. ${ }^{15,16}$ Hence, it is desirable to be able to directly measure the electrical properties of the tunnel junction and validate the effectiveness of the growth process.

Off-axis electron holography (from now on referred to as electron holography) is a TEM based technique that can be used to measure the electrostatic potentials in semiconductor devices with $\mathrm{nm}$-scale resolution. ${ }^{17,18}$ Here an object wave that passes through a region of interest is interfered with a reference wave that passes through vacuum by using an electron biprism to form an interference pattern known as a hologram. By using a simple Fourier reconstruction procedure, the phase and amplitude of the electrons that have passed through the specimen can be recovered. Then in the absence of a magnetic field, or from dynamical diffraction which strongly affects the phase of the electrons, the total electrostatic potential can be obtained, where $\mathrm{V}_{\text {dopant }}$ is the dopant potential, $\mathrm{V}_{\text {piezo }}$ is the piezo-electric polarization and $\mathrm{V}_{\mathrm{MIP}}$ is the mean inner potential. The mean inner potential is related to the volume averaged density of electrons in a material. ${ }^{19}$

$$
\Delta \Phi=C_{E} \int_{0}^{t}\left(V_{\text {dopant }}+V_{\text {piezo }}+V_{M I P}\right) d z
$$

The total measured phase is the integral of the potential measured through the specimen of thickness, $t$. The constant $C_{E}$ is equal to $0.0073 \mathrm{rad} \cdot \mathrm{nm}^{-1} \cdot \mathrm{V}^{-1}$ for $200 \mathrm{kV}$ electrons. ${ }^{18}$ The $\mathrm{V}_{\text {MIP }}$ has been calculated to be $16.8 \mathrm{~V}$ for $\mathrm{GaN}$ from density functional theory ${ }^{19}$ and this will remain a constant across these homogenous GaN specimens if perfectly parallel-sided specimens are examined. Small variations of specimen thickness will lead to very large changes in phase. For example, a $10 \mathrm{~nm}$ thickness variation will lead to a change in measured phase of 1.2 rads in GaN which is the same order of magnitude as would be expected across a doped junction. Thus, the FIB becomes an important tool as high-quality parallel-sided specimens can be produced. By combining state-of-the-art electron microscopes, advanced reconstruction procedures and the summation of stacks of electron holograms ${ }^{20,21}$ it is possible to obtain maps of the electrostatic potential in nitride based semiconductor devices with spatial resolutions as good as $1 \mathrm{~nm}$ and an excellent signal to noise ratio. As such, these recent developments mean that it is possible to routinely measure the depletion width of the TJ specimens.

In this paper, a systematic study of off-axis electron holography analysis combined with secondary ion mass spectrometry (SIMS) and opto-electrical testing is shown. These techniques have been correlated to better understand the influence that the doping interface and polarization engineering have on the electrical behavior of several GaN tunnel junctions developed by all-metalorganic vapour-phase epitaxy 
(MOCVD) and hybrid molecular-beam epitaxy (MBE) / MOCVD growths on top of InGaN/GaN blueemitting LED structures. These experiments are optimized for dopant profiling and similar device studies focused on the measurements of the polarization fields in quantum wells are shown elsewhere. ${ }^{21}$

\section{Specimen Growth}

Figure 1 shows schematics of the TJs that were studied here. The base LED structures were grown at the same time in a 7x2 inch Aixtron AIX6 shower-head MOCVD reactor. Epitaxial growth was then performed in a different reactor for the deposition of the $n$-doped components of the TJ layers. The first TJs (TJ-A and TJ-B) were grown in a home-made MOCVD vertical reactor (HMR) using Trimethylgallium (TMGa), triethylgallium (TEGa), trimethylindium (TMIn), trimethylaluminium (TMAI), bis(cyclopentadienyl)magnesium $\left(\mathrm{Cp}_{2} \mathrm{Mg}\right)$, silane $\left(\mathrm{SiH}_{4}\right)$ and ammonia $\left(\mathrm{NH}_{3}\right)$ as the precursors for $\mathrm{Ga}$, In, $\mathrm{Al}, \mathrm{Mg}, \mathrm{Si}$, and $\mathrm{N}$, respectively. The growth temperatures were measured by pyrometry using a reflectivity corrected system from Laytec.

The LED structure comprised a $2 \mu \mathrm{m}$ non-intentionally-doped GaN template, $2 \mu \mathrm{m}$ of n-doped GaN and 5 $\left[\mathrm{In}_{0.15} \mathrm{Ga}_{0.85} \mathrm{~N}(2 \mathrm{~nm}) / \mathrm{GaN}(12 \mathrm{~nm})\right]$ quantum wells (QWs), followed by a $20 \mathrm{~nm} \mathrm{Al}_{0.15} \mathrm{Ga}_{0.85} \mathrm{~N}$ electronblocking-layer (EBL), 100nm p-GaN and a 10nm p++ cap of GaN. At this stage the p-type doping was activated by an in-situ anneal under $\mathrm{N}_{2}$ atmosphere during 20 minutes at a temperature of $700^{\circ} \mathrm{C}$. Then, for TJ-A, a $20 \mathrm{~nm}$ n++ GaN layer was grown, followed by $200 \mathrm{~nm} n+\mathrm{GaN}$ and the $20 \mathrm{~nm} \mathrm{n}++\mathrm{GaN}$ capping layer. TJ-B used the same stack except for the insertion of a $4 \mathrm{~nm} \ln 0.1 \mathrm{Ga}_{0.9} \mathrm{~N}$ interlayer. All the $\mathrm{n}$-doped parts of the TJs were grown using $\mathrm{N}_{2}$ as the carrier gas, $800^{\circ} \mathrm{C}$ as the growth temperature, $100 \mathrm{mbar}$ pressure and $2.5 \mu \mathrm{m} / \mathrm{h}$ growth rate in order to minimize the thermal budget and to reduce the Mg acceptor repassivation by hydrogen. ${ }^{22}$

The $\mathrm{n}$-doped component of the hybrid TJs $\mathrm{C}$ and D were grown in a Riber $32 \mathrm{MBE}$ reactor equipped with Si and Ge solid sources and using $\mathrm{NH}_{3}$ as a N precursor. For TJ-C, the first layer consisted of $20 \mathrm{~nm}$ of $\mathrm{n++}$ GaN $\left([\mathrm{Si}]=1 \times 10^{20} \mathrm{~cm}^{-3}\right), 200 \mathrm{~nm}$ of $\mathrm{n}+\mathrm{GaN}\left([\mathrm{Si}]=1 \times 10^{19} \mathrm{~cm}^{-3}\right)$ and the final cap of $20 \mathrm{~nm}$ of $\mathrm{n}++\mathrm{GaN}([\mathrm{Si}]=$ $\left.1 \times 10^{20} \mathrm{~cm}^{-3}\right)$, while for TJ-D the stacking consisted of $20 \mathrm{~nm}$ of $\mathrm{n}++\mathrm{GaN}: \mathrm{Ge}\left([\mathrm{Ge}]=5 \times 10^{20} \mathrm{~cm}^{-3}\right)$, followed by $200 \mathrm{~nm}$ of $\mathrm{n}+\mathrm{GaN}: \mathrm{Ge}\left([\mathrm{Ge}]=1 \times 10^{19} \mathrm{~cm}^{-3}\right) \cdot{ }^{12}$ The purpose of using Ge as the dopant in for TJ-D was that higher doping levels are possible due to the reduction of tensile strain. In addition, it has been shown that $\mathrm{Ge}$ is less prone to nitridation than $\mathrm{Si}$, which might be an issue for the cells used in $\mathrm{NH}_{3} \mathrm{MBE}$ since it could limit the Si flux and thus the doping levels. ${ }^{23}$

In all cases, the samples were exposed to air during the transfer from the shower-head growth reactor to the HMR or MBE reactors. No chemical treatment was performed as it is believed that impurities absorbed during transfer, such as oxygen might contribute to the insertion of mid-gap states in the depletion region and improve the tunneling behavior. ${ }^{24-26}$

The LEDs were fabricated using standard photolithography and reactive ion etching to produce mesas of (100x100) $\mathrm{mm}^{2}$. The metallic contacts comprised Ti/Al/Ni/Au stacks with thicknesses of 30/180/40/200 
$\mathrm{nm}$ respectively. Then electrical characterization (I-V curves) were performed in continuous wave conditions at room temperature using a Keithley 2104 sourcemeter. The electroluminescence was measured using a BWTek spectrometer.

\section{Characterisation Methods}

Parallel-sided and flat specimens for examination by electron holography were realized using FIB milling in a FEI Strata 400. Rough milled specimens containing the region of interest were attached to a grid using in situ lift-out and then a range of relatively thick electron transparent windows were prepared in a single specimen (250-400 nm) using $30 \mathrm{kV}$ ions. Finally cleaning was performed on each side using $2 \mathrm{kV}$ ions. As the specimens are parallel sided and homogenous in the TJ region, Equation 2 describes how the step in phase can be related to the $\mathrm{V}_{\text {dopant }}$ and $\mathrm{V}_{\text {piezo. }}$. Under these conditions, the change in phase can be described as follows:

$$
\Delta \Phi=C_{E} \times\left(V_{\text {dopant }}\left(t_{\text {cryst }}-t_{\text {inactive }}\right)+V_{\text {piezo }} t_{\text {cryst }}\right)
$$

where $t_{\text {cryst }}$ is the crystalline thickness of the specimen. The inactive thickness, $t_{\text {inactive }}$ is a modified crystalline surface region which does not contribute towards the measured phase change arising from the active dopants. This is caused by a combination of structural damage caused by FIB milling, which can trap the dopant atoms in defect states and the effect of surface charging or pinning which leads to band bending at the specimen surfaces. ${ }^{27}$ At this time the inactive layer in GaN is poorly understood, although experimental measurements published elsewhere show that the step in potential measured across GaN pn junctions is much less than theory. ${ }^{27,28}$ Consequently, the specimens examined in this study were left intentionally thick to reduce the effect of the inactive layer and care was taken to keep the experimental conditions identical for each device such that the results could be compared relatively. At this time it is assumed that there is no (or negligible) tinactive for the piezoelectric field. This assumption has not been proven experimentally and has been only been confirmed by 3D simulations that include charging on the specimen surfaces. ${ }^{30}$

Electron holograms were acquired using the FEI Titan Ultimate operated at $200 \mathrm{kV}$. The holograms were recorded with a fringe spacing of $1.2 \mathrm{~nm}$ using a Gatan OneView 4k camera which provided a large field of view. To improve the spatial resolution, the image corrector was used to improve the resolution of the Lorentz lens to less than $1 \mathrm{~nm}$. The electron holograms were then reconstructed to provide phase images with a spatial resolution of $2.4 \mathrm{~nm}$. For these holograms with fine fringe spacing, the fringe contrast was less than would be expected for wider fringes. This is due to the limited coherence of the beam and the need to sample the fringes using the finite number of CCD pixels. This loss of contrast was compensated for by acquiring a stack of 32 electron holograms, each acquired for $8 \mathrm{~s}$ which were summed using the Holoview software. ${ }^{20}$ Figure $2(a)$ and (b) show reconstructed phase images acquired from a single hologram and from a summed series respectively. An additional interest of adding a stack of holograms together is that thick GaN samples can be examined using $200 \mathrm{kV}$ electrons as opposed to $300 \mathrm{kV}$, and relatively low beam currents can be used which has the effect of reducing the beam damage. ${ }^{20}$ The 
improvement in the signal to noise in these phase images is clear. To quantify the improvement, a standard deviation of the region below the QWs can be measured. The phase image reconstructed from a single hologram gives a phase resolution of $2 \pi / 13$ compared to $2 \pi / 74$ for the reconstruction from the stack. Indeed, for the phase reconstruction from the stack of holograms, the effect of specimen thickness variations arising from different FIB milling depths due to the rough specimen surfaces can be seen in the form of a curtaining effect across the phase. As mentioned previously, the rough specimen surface is due to the low temperature growth. These artefacts could be avoided by using back side FIB milling if required. The equivalent reconstructed amplitude images are shown in Figures 2(c) and (d) where a single hologram and a stack of 32 holograms have been used respectively. Careful examination of the amplitude images is an important step when performing electron holography as they indicate the presence of dynamical diffraction. In electron holography it is assumed that the sample is a weak phase object where the change in phase is much stronger than the change in amplitude. For the thick specimens that are examined here, clearly this will not be true. However, for these types of experiments where the relative phase is measured across a region of interest, the specimen is tilted until the amplitude images have a homogenous contrast across them. In the examples shown here, as there are no strong contrast changes in the region of interest, the phase can be interpreted.

A phase profile extracted from the indicated region is shown in Figure 2(e). As the LEDs have contributions from $V_{\text {MIP }}, V_{\text {dopants }}$ and $V_{\text {piezo, }}$ it is important to discuss the contribution of the different regions to the measured phase.

1. Here the phase is flat due to constant n-doped GaN. The low concentration of dopants means that the inactive thickness dominates and only the $\mathrm{V}_{\text {MIP }}$ of $16.9 \mathrm{~V}$ is detected. ${ }^{19}$

2. The region containing the InGaN QW structures has two components, the negative phase arising from the $\mathrm{V}_{\text {piezo }}$ and the positive phase from the change in MIP from the InGaN. ${ }^{30}$ DFT simulations of InN suggest that the $\mathrm{V}_{\text {MIP }}$ is $18.9 \mathrm{~V} .{ }^{19}$ As such we expect an increase of $0.2 \mathrm{~V}$ for each increase of $10 \%$ In in the InGaN QW structures.

3. The AIGaN electron blocking layer shows a reduction in phase as the MIP of AIN is $15.9 \mathrm{~V} .{ }^{19}$ As such a decrease in $0.1 \mathrm{~V}$ is expected for each increase of $10 \% \mathrm{Al}$ in the $\mathrm{AIGaN}$ layers.

4. In the $\mathrm{p}$-doped layer, despite the $\mathrm{Mg}$ concentration being in the $1 \times 10^{19} \mathrm{~cm}^{-3}$ range, the negative dopant contrast is difficult to observe due to the inactive layer in the specimen and low ionized dopant concentration at room temperature.

5. In the TJ region, dark contrast arising from the heavily doped p++ is now observable. Interpretation of this region is complicated due to the presence of the InGaN inter-layer where phase changes arising from both the $\mathrm{V}_{\text {MIP }}$ and $\mathrm{V}_{\text {piezo }}$ are also present.

6. The heavily $n$-doped region in the TJ shows a strong positive phase change.

7. The lightly n-doped region does not show strong dopant contrast due to the inactive thickness.

8. This $n$-doped region is uninterpretable as the crystalline quality of the top of the specimen leads to strong dynamical diffraction which strongly modifies the phase. 
If the crystalline thickness of the specimen is accurately measured, then potential can then be calculated directly from the phase using Equation 2. Here two-beam convergent beam electron diffraction (CBED) is used which is accurate to +/- $5 \mathrm{~nm}$ for these GaN specimens. As previously discussed, the calculated potential is significantly less than expected. For the purpose of this work, the inactive thickness is unknown and as such it is not possible to accurately quantify the potentials. The calculated potential has been presented here, as opposed to the measured phase out of general interest to illustrate the problems with quantification. However, the main focus of this work is not the absolute measurement of the active dopant concentration, but the study of the TJ width and therefore it is the spatial resolution of the phase images that is important.

\section{Spatial Resolution of Holography Experiments}

Figure 3(a) shows a HAADF STEM image of the region containing the QW structures in TJ-B acquired using a probe corrected microscope at atomic resolution with the specimen oriented on the zone axis. This can be compared to the amplitude image of the same region acquired from the same sample shown in Figure 3(b). Here, to reduce the dynamical diffraction the specimen has been rotated from the (0120) zone axis around the $\{0001\}$ growth direction whilst taking care to keep the layers parallel to the beam such that information is not lost in projection through the specimen. The slightly dark lines either side of the QWs arise from diffraction contrast at the GaN/InGaN/GaN interfaces. From the HAADF STEM images, the average width of the QW structures is $2.2 \mathrm{~nm}$, whereas from the reconstructed amplitude $3.0 \mathrm{~nm}$ is measured. Thus despite the spatial resolution of the reconstructed electron holograms being $2.4 \mathrm{~nm}$ and the specimens being tilted from a zone axis for examination, measurement of the width of the QW structures is overestimated by only $0.8 \mathrm{~nm}$. As such, if the experimental parameters can be controlled the spatial resolution of the electron holography as performed here is an appropriate tool for the measurement of the width of the TJs. It is possible to use advanced reconstruction methods such as double exposure holography ${ }^{30,31}$ or phase change holography ${ }^{32}$ in order to improve the spatial resolution. However, these approaches were not implemented here.

\section{Removal of the Mean Inner Potential}

To compare the step in potential across the different TJs, it is necessary to accurately remove the contribution of the MIP from the InGaN interlayer in the TJ-B. The targeted properties were for a QW width of $2.0 \mathrm{~nm}$ and an In concentration of $16 \%$ to provide blue emission. For the interlayer in the TJ, the targeted In concentration was $10 \%$. The large interaction volume of the electron beam compared to the size of the QWs makes accurate quantification of the In concentration by EDX difficult. ${ }^{34}$ As such geometrical phase analysis (GPA) was used to measure the deformation in the InGaN QW layer to provide a measure of the In content. Figure 4(a) shows a HRSTEM image of the 5 InGaN QW structures showing an excellent crystalline structure with an average width of $2.2 \mathrm{~nm}$. A GPA algorithm was applied to the HAADF STEM image. ${ }^{33}$ Figure $4(\mathrm{~b})$ shows the deformation map for the (0001) growth direction. As the InGaN QW layers are grown epitaxially on the GaN substrate layers, they will be relaxed in the growth 
direction and as such the In concentration can be determined from the deformation. ${ }^{30}$ As a comparison, EDX measurements were also performed and these results are shown in Figure 4(c). These were performed using a FEI Titan Themis operated at $200 \mathrm{kV}$ equipped with the Super-X detection system. The growth direction deformation profiles are shown in Figure 4(d). Here an average value of $2.6+/-0.2 \%$ is measured which corresponds to an In concentration of $17.3+/-1.5 \%$. Due to the high doping concentration, it was not possible to perform GPA across the InGaN interlayer as the quality of the crystal and hence the atomic resolution HAADF images was not sufficiently high. To be able to estimate the In concentration of the interlayer, EDX was then performed and the relative In concentration between the InGaN QWs and the interlayer were compared. Figure 4(e) shows the In profile obtained from the EDX experiments, here the intensity of the In interlayer signal is $70 \%$ of the QWs which corresponds to a In concentration of 10 +/- $1.5 \%$ calibrated from the GPA measurements which is consistent with the expected value.

From the estimated In concentrations it was then possible to remove their MIP contribution from the measured potential. To achieve this, the MIP for the InGaN layers was simulated using a linear relationship between the values of $16.9 \mathrm{~V}$ for $\mathrm{GaN}$ and $18.9 \mathrm{~V}$ for $\mathrm{InN}$. The simulated InGaN layers were then convolved using a Gaussian function to account for the spatial resolution of the electron holography experiments. Figure 5 shows the simulated MIP profiles along with the experimentally measured potential and the potential with the In MIP component removed. The experimental profiles are very sensitive to the removal of the MIP and as such this is an accurate way to determine the In concentration in the materials. Although these experiments were not optimized to measure the piezoelectric potential in the QW region, especially regarding the specimen thickness, they act as test to see if the MIP is correctly removed from the total potentials. For four of the QWs, the best fitting MIP profiles use values of $0.34 \mathrm{~V}$ which corresponds to an In concentration of $17 \%$. In a single layer, a value of $0.25 \mathrm{~V}$ is used, corresponding to an In concentration of $12.5 \%$. The values of $17 \%$ are consistent with both the measurement of deformation and the emission of the LED $(440 \mathrm{~nm})$. Having validated this approach, a value of $10 \%$ In has been used to remove the MIP in the TJ region and provide an improved measurement of the electrostatic potential in the TJ.

\section{Chemical Mapping}

The $\mathrm{Si}, \mathrm{Ge}$, In and $\mathrm{Mg}$ concentration profiles were measured by SIMS analysis through multiple measurements with optimized relative ion yields because of the different chemical nature of these atoms. The doping profiles were calculated from the obtained concentration values.

For the Ge doped TJ-LED D structure it was possible to gain further insight into the dopant distribution from electron dispersive X-ray spectrometry (EDX) to measure the distribution of the Ge in the LEDs. For the other LED structures, it was not possible to use EDX as the Si concentration of $1 \times 10^{19} \mathrm{~cm}^{-3}$ is undetectable in our TEM at this time. The higher $\mathrm{Mg}$ dopant concentrations of $1 \times 10^{20} \mathrm{~cm}^{-3}$ are difficult to detect by EDX as the small signal from the Mg K-line $(1.25 \mathrm{keV})$ is next to the high background from the 
Ge L-line (1.19 keV). For Ge it is possible to measure the L-edge at $9.85 \mathrm{keV}$ which allows more easy detection of these dopants.

\section{Characterization of the Tunnel Junctions}

Figure 6 shows the SIMS profiles for the different LED structures. Importantly, the Mg and Si profiles are not obtained during the same analysis as they are not detected in the same mode, being negative mode for Si and positive mode for both Mg and In. Therefore, in Figures (a), (b) and (c) the Mg and Si profiles are superimposed in the same figure by measuring the SIMS craters and reconstructing the profiles, which obviously may lead to errors in the relative positioning. ${ }^{15}$ For the case of $\mathrm{Ge}$, it can be detected in both modes and was detected in positive mode here, such that the $\mathrm{Ge}$ and $\mathrm{Mg}$ are well aligned.

Figures 6(a) and (b) show the SIMS profiles of TJ-A and TJ-B grown by MOCVD. We observe that the Mg profile is not sharp and extends in the Si doped region. This can be interpreted in terms of segregation of the excess $\mathrm{Mg}$ that is accumulated during the p-type layer growth. In addition, the top n-type layers grown at $800^{\circ} \mathrm{C}$ were rough $(\mathrm{rms}>10 \mathrm{~nm})$ which has the effect of broadening the spatial resolution of the SIMS profiles. During the MOCVD growth of the Si n-doped top region of the TJ, the Mg is also incorporated into the GaN. These $\mathrm{Mg}$ atoms can be passivated by hydrogen and although the growth is performed under $\mathrm{N}_{2}$ gas, $\mathrm{H}_{2}$ is present due to the decomposition of ammonia and organometallics. The passivated $\mathrm{Mg}$ has a lower formation energy than $\mathrm{Mg}$ and as such is more likely to appear than substitutional dopants. ${ }^{35,36}$ Thus, the Mg incorporated during the growth of the Si doped layers in MOCVD can be expected to be in large part passivated.

SIMS profiles of the MBE grown TJ-C and TJ-D show much sharper Mg profiles at higher concentrations. As the underlying LED structure is the same for all samples, the Mg peak amplitude in the $\mathrm{p}$ type region should be identical. There are two explanations for the difference between TJs A-B and C-D. The first is the diffusion and segregation of $\mathrm{Mg}$ that is present in the MOCVD growth at $800^{\circ}$ and absent in the MBE growth at $720^{\circ} \mathrm{C}$. The second is the much larger roughness of TJs A-B when compared to TJs C-D, which tends to broaden the SIMS profiles. This second effect seems limited as the InGaN interlayer remains quite well defined as shown in Figure 4(b). The same remark holds for the Si doping, which is sharper in MBE TJ-C than in TJ-A and TJ-B. The Ge dopant profile is also sharp in the MBE-grown TJ-D. These interpretations are equally applicable to the Si profiles.

The SIMS profiles can be used to model the electrostatic potentials in the TJs. These calculations were made with nextnano. ${ }^{37}$ For TJ-A and TJ-B, the calculation based on the Mg profiles from Figures 6(a) and (b) leads to the conclusion that the junction is located far from its expected location which is where the $\mathrm{Mg}$ and Si profiles cross. The junction width is calculated to be about $20 \mathrm{~nm}$ which is not consistent with observations made by electron holography and the electrical measurements. Such a wide junction would have a negligible tunnel transparency which is contrary to the electrical measurements. ${ }^{22}$ To provide more accurate simulations by removing the effects of surface roughness on the measured SIMS profiles, for TJ$A$ and $B, a$ Mg profile was used with an exponential decrease similar to those observed for TJ-C and D. 
The Si or Ge profile was extracted directly from the SIMS for all four LEDs. The results of the calculations are shown in Figure 7. Instead of plotting the conduction band energy, the opposite value is shown to provide a quantity varying as the potential. The profiles are offset by $5 \mathrm{~nm}$ for clarity. The width of the tunnel junction can be estimated from the width of the depletion region. Here measurements $15,10,8$ and $7 \mathrm{~nm}$ can be obtained for TJs A, B, C and D respectively. For the TJ-B profile, the InGaN interlayer can be clearly seen.

The results from the electron holography for the LEDs grown using Si n-type dopants are shown in Figure 8. Figure 8(a) shows a HAADF STEM image, (b) potential map and (c) potential profile for TJ-A which was grown using all MOCVD at low temperature. Here a TJ of $15+/-2 \mathrm{~nm}$ is measured. This is in good agreement with the simulated value shown in Figure 7. Figure 8(d) shows a HAADF STEM image, (e) potential map and (f) the potential profile for TJ-B that was grown at low temperature and incorporates an InGaN interlayer. A TJ width of $8+/-2 \mathrm{~nm}$ is measured which is significantly less than the one measured for TJ-A, and in again in agreement within error to the simulated value. Finally, Figure 8(g) shows a HAADF STEM image, (h) potential map and (i) potential profile for the hybrid TJ-C that was grown using both MOCVD and MBE. For this LED, a TJ of $10+/-2 \mathrm{~nm}$ is measured, again in agreement with the simulated value. For the holography experiments, eliminating the dynamical diffraction in these thick specimens is key, as such it is extremely difficult to have a perfectly clean phase image across the whole LED structure. As such, for these experiments the diffraction condition in the TJ region was optimized and, in some cases, the regions containing the InGaN QW structures are not interpretable due to dynamical diffraction contrast.

Figure 9 shows the experimental results for TJ-D which was grown using Ge as the n-type dopant. Figure 9(a) shows a HAADF STEM image of the device and (b) a potential map. The profile measured across the TJ region is shown in Figure 9(c) and a width of $9+/-2 \mathrm{~nm}$ is measured. This is in agreement to within experimental error with the simulated value shown in Figure 7. Figures 9(d) shows an EDX map for Al, (e) In and (f) Ge respectively. A profile showing the Ge concentration measured across the TJ region is shown in Figure 9(g). Although this region is supposed to be of constant dopant concentration, two distinct peaks can be observed in both the EDX and also in the potential map measured by electron holography.

The current-voltage and electro-luminescence characteristics for all four samples is shown in Figure 10. ${ }^{12,22}$ To estimate the electrical performances of the different samples the LED voltages were compared at a current density of $100 \mathrm{~A} / \mathrm{cm} 2$. From Figure $10(\mathrm{a})$ it can be seen that $10.8,7.1,4.5$, and $5.4 \mathrm{~V}$ were measured for samples TJA, TJB, TJC, and TJD, respectively. These values are somewhat higher that can be found in the recent literature, especially for full MOCVD structures for which voltages as low as $3.5 \mathrm{~V}$ at $100 \mathrm{~A} / \mathrm{cm} 2$ have been demonstrated. To achieve this high performance, selective area regrowth was used to minimize the issue of $\mathrm{H}$ re-passivation of the $\mathrm{Mg}$ acceptors during the top $\mathrm{n}-\mathrm{GaN}$ growth of the tunnel junction. ${ }^{[38]}$ Indeed, the low temperature approach used for our full-MOCVD tunnel junction LEDs could be improved by increasing the $n$-type doping of the $n++$ part of the tunnel junction. Regarding the hybrid 
approaches combining MBE and MOVCD our results are close to the best reported results giving a voltage of $4 \mathrm{~V}$ at $100 \mathrm{~A} / \mathrm{cm} 2 .^{[39]}$

The higher current densities in TJ-C and D are in qualitative agreement with the observed TJ widths given by the electron holography measurements and SIMS profiles. The higher active dopant concentrations at the junctions are expected to increase the tunneling probability through the narrowing of the TJ width. The MOCVD tunnel junctions are more resistive than the hybrids for all voltages measured, which is also consistent with the TJ width suggested by the holography method since TJ-A has the widest TJ. This value is considerably reduced by adding an InGaN interlayer, which helps TJ-B to obtain a junction as thin as in the hybrid samples. However, since the I-V characteristics depend not only on the tunneling widths but on several other parameters such as series and contact resistances, TJ-B remains more resistive than its hybrid counterparts TJ-C and TJ-D. Evidence of higher resistivity can be observed in Figure 10(b) which shows current crowding around the top electrical contact in the optical measurement for TJ-B. ${ }^{40} \mathrm{An}$ additional parameter that has not been exploited here is the measured step in potential and the value of $0.36+/-0.05 \mathrm{~V}$ that is measured for TJ-B is much less than the relatively high values of 1.5 and $1.29+-/$ $0.05 \mathrm{~V}$ that is measured for TJ-C and D. These large differences may be due to improved dopant activity in the specimens grown by MBE. However, TJ-B is also the thinnest of the different TEM lamellas observed, with a crystalline thickness of $240 \mathrm{~nm}$. This would result in the inactive thickness having a larger effect compared to the other TJs. ${ }^{29}$ Although these results were reproducible on different specimens prepared from the same wafers, more work is required to assess parameters such as specimen preparation and charging if we wish to correctly interpret the measured step in potentials for these GaN-based devices.

Even though a valuable qualitative insight on the electrical characteristics of the diodes has been obtained, the spatial resolution of both SIMS and holography do not allow for a precise quantitative prediction of the behavior of the diodes. This is mainly due to the fact that these measurements are linearly dependent on the tunnel junction width, while the current depends exponentially on it. An additional reason is that the current also varies with the bandgap energy in the TJ, while SIMS and electron holography cannot quantitatively measure it. However, as the holography provides a direct measurement of TJ width, it can provide insight for the interpretation of the electrical results. In the case of TJs, where the junction interface plays a vital role to the performance of the device, electron holography allows the assessment of the local active doping which SIMS cannot do, in particular for rough surfaces.

Advances in the stability of FIBs and TEMs mean that it is possible to perform reproducible off-axis electron holography experiments over a long time period. However, we admit that it is challenging to apply this technique to semiconductor specimens that contain high densities of defects and dislocations. These lead to dynamical diffraction sources which provide misleading information about the potentials carried by the electron phase. A solution to this problem is to prepare large specimens which are $20-30$ 
microns in length and contain different thickness regions, of which there is a better chance of finding a region of good crystal quality to perform the experiments.

\section{Conclusion}

Off-axis electron holography has been used to measure the width of a series of TJ-LED devices with simultaneous high sensitivity and a spatial resolution of $2.4 \mathrm{~nm}$. The LEDs were grown using identical MOCVD conditions to the bottom $\mathrm{p}$-doped layer of the TJ. The top $\mathrm{n}$-type parts of the TJs were then grown by low temperature MOCVD in the case of TJ-A and B, or by MBE in the case of TJ C and D. The objective of this work was to use electron holography to provide information about the active dopant distribution and link this knowledge to the opto-electrical performances of the devices. This is complicated as the information about the dopant potentials is mixed with contributions from the mean inner potential and as such these components need to be unmixed. To achieve this, the In concentration in the interlayer in TJ-B has been estimated by deformation mapping which is also consistent with the mean inner potential obtained by holography. As such, it was possible to quantitatively measure the TJ-widths by electron holography and these values were consistent with simulations that were performed using the SIMS data.

The performance of the devices are not only sensitive to the TJ-width. However, the current density measurements, the simulations and holography results are largely consistent with each other. TJ-A with the widest junction has the poorest electrical characteristics, whereas the MBE-grown TJs C and D have narrower junctions and the best electrical characteristics. The outlier is TJ-B which from only the electron holography data, good electrical properties would be expected, however the optical measurements in this case explain the poor performance. In summary, we have shown that from the use of the latest generation of transmission electron microscopes and advanced data processing, off-axis electron holography is a suitable experimental technique for the measurement of the width of tunnel junctions in GaN-based LED devices.

\section{Acknowledgements}

This work, done on the NanoCharacterisation PlatForm (PFNC), was supported by the "Recherches Technologiques de Base" Program of the French Ministry of Research. We would like to acknowledge GaNeX (No. ANR-11-LABX-0014) and the French National Research Agency (ANR) for funding this research through the DUVET project (No. ANR-17-CE08-0024).

\section{Data Availability Statement}

The data that support the findings of this study are available from the corresponding author upon reasonable request.

\section{References}


${ }^{1}$ F. Akyol, S. Krishnamoorthy, and S. Rajan, Appl. Phys. Lett. 103, 081107 (2013).

2 E.C. Young, B.P. Yonkee, F. Wu, S.H. Oh, S.P. DenBaars, S. Nakamura, and J.S. Speck, Appl. Phys. Express 9, 022102 (2016).

${ }^{3}$ S.-R. Jeon, Y.-H. Song, H.-J. Jang, G.M. Yang, S.W. Hwang, and S.J. Son, Appl. Phys. Lett. 78, 3265 (2001).

${ }^{4}$ H. Hirayama, N. Maeda, S. Fujikawa, S. Toyoda, and N. Kamata, Jpn. J. Appl. Phys. 53, 100209 (2014).

${ }^{5}$ Y. Nagasawa and A. Hirano, Appl. Sci. 8, 1264 (2018).

${ }^{6}$ M. Shatalov, W. Sun, R. Jain, A. Lunev, X. Hu, A. Dobrinsky, Y. Bilenko, J. Yang, G.A. Garrett, L.E. Rodak, M. Wraback, M. Shur, and R. Gaska, Semicond. Sci. Technol. 29, 084007 (2014).

${ }^{7}$ M. Saha, A. Biswas, and H. Karan, Opt. Mater. 77, 104 (2018).

${ }^{8}$ S.J. Kowsz, E.C. Young, B.P. Yonkee, C.D. Pynn, R.M. Farrell, J.S. Speck, S.P. DenBaars, and S. Nakamura, Opt. Express 25, 3841 (2017).

${ }^{9}$ D. Kasahara, D. Morita, T. Kosugi, K. Nakagawa, J. Kawamata, Y. Higuchi, H. Matsumura, and T. Mukai, Appl. Phys. Express 4, 072103 (2011).

${ }^{10}$ Y. Zhang, S. Krishnamoorthy, J.M. Johnson, F. Akyol, A. Allerman, M.W. Moseley, A. Armstrong, J. Hwang, and S. Rajan, Appl. Phys. Lett. 106, 141103 (2015).

${ }^{11}$ Y. Zhang, S. Krishnamoorthy, F. Akyol, A.A. Allerman, M.W. Moseley, A.M. Armstrong, and S. Rajan, Appl. Phys. Lett. 109, 121102 (2016).

${ }^{12}$ V. Fan Arcara, B. Damilano, G. Feuillet, S. Vézian, K. Ayadi, S. Chenot, and J.-Y. Duboz, J. Appl. Phys. 126, 224503 (2019).

${ }^{13}$ F. Akyol, Y. Zhang, S. Krishnamoorthy, and S. Rajan, Appl. Phys. Express 10, (2017).

${ }^{14}$ E. Di Russo, A. Mavel, V. Fan Arcara, B. Damilano, I. Dimkou, S. Vézian, A. Grenier, M. Veillerot, N. Rochat, G. Feuillet, B. Bonef, L. Rigutti, J.-Y. Duboz, E. Monroy, and D. Cooper, Nanotechnology 31, 465706 (2020).

${ }^{15}$ Y. Kuwano, M. Kaga, T. Morita, K. Yamashita, K. Yagi, M. Iwaya, T. Takeuchi, S. Kamiyama, and I. Akasaki, Jpn. J. Appl. Phys. 52, 08JK12 (2013).

${ }^{16}$ S. Neugebauer, M.P. Hoffmann, H. Witte, J. Bläsing, A. Dadgar, A. Strittmatter, T. Niermann, M. Narodovitch, and M. Lehmann, Appl. Phys. Lett. 110, 102104 (2017).

${ }^{17}$ H. Lichte and M. Lehmann, Rep. Prog. Phys. 71, 016102 (2007).

${ }^{18}$ A. Tonomura, Rev. Mod. Phys. 59, (1987).

${ }^{19}$ P. Kruse, M. Schowalter, D. Lamoen, A. Rosenauer, and D. Gerthsen, Ultramicroscopy 106, 105 (2006).

${ }^{20}$ V. Boureau, R. McLeod, B. Mayall, and D. Cooper, Ultramicroscopy 193, 52 (2018).

${ }^{21}$ D. Cooper, V. Boureau, A. Even, F. Barbier and A. Dussaigne, Nanotechnology, 31, 475705 (2020).

${ }^{22}$ V. Fan Arcara, B. Damilano, G. Feuillet, A. Courville, S. Chenot, and J.-Y. Duboz, AIP Adv. 9, 055101 (2019).

${ }^{23}$ M. Mukhopadhyay, S.K. Ray, and C.K. Maiti, J. Vac. Sci. Technol. B Microelectron. Nanometer Struct. Process. Meas. Phenom. 14, 1682 (1996).

${ }^{24}$ E. Vadiee, E.A. Clinton, H. McFavilen, A.S. Weidenbach, Z. Engel, C. Matthews, C. Zhang, C. Arena, R.R. King, C.B. Honsberg, and W.A. Doolittle, Appl. Phys. Express 11, 082304 (2018). 
${ }^{25}$ J.M.O. Zide, A. Kleiman-Shwarsctein, N.C. Strandwitz, J.D. Zimmerman, T. Steenblock-Smith, A.C. Gossard, A. Forman, A. Ivanovskaya, and G.D. Stucky, Appl. Phys. Lett. 88, 162103 (2006).

${ }^{26}$ S. Krishnamoorthy, T.F. Kent, J. Yang, P.S. Park, R.C. Myers, and S. Rajan, Nano Lett. 13, 2570 (2013).

${ }^{27}$ L. Amichi, I. Mouton, V. Boureau, E.D. Russo, P. Vennéguès, P.D. Mierry, A. Grenier, P.-H. Jouneau, C. Bougerol, and D. Cooper, Nanotechnology 31, 045702 (2019).

28 J.B. Park, T. Niermann, D. Berger, A. Knauer, I. Koslow, M. Weyers, M. Kneissl, and M. Lehmann, Appl. Phys. Lett. 105, 094102 (2014).

${ }^{29}$ P.K. Somodi, A.C. Twichett-Harrison, P.A. Midgley, B.E. Kardynal, C.H.W. Barnes, and R.E. DuninBorkowski, Ultramicroscopy 134, 160 (2013).

${ }^{30}$ V. Boureau and D. Cooper, J. Appl. Phys. 128, 155704 (2020).

${ }^{31}$ V.V. Volkov, M.G. Han, and Y. Zhu, Ultramicroscopy 134, 175 (2013).

${ }^{32}$ Q. Ru, G. Lai, K. Aoyama, J. Endo, and A. Tonomura, Ultramicroscopy 55, 209 (1994).

${ }^{33}$ M. Hytch, J.L. Putaux, and J.M. Pénisson, Nature 423, 270 (2003).

${ }^{34}$ P. Rueda-Fonseca, E. Robin, E. Bellet-Amalric, M. Lopez-Haro, M. Den Hertog, Y. Genuist, R. André, A. Artioli, S. Tatarenko, D. Ferrand, and J. Cibert, 16, 1637 (2016).

${ }^{35}$ S. Brochen, J. Brault, S. Chenot, A. Dussaigne, M. Leroux, and B. Damilano, Appl. Phys. Lett. 103, 032102 (2013).

${ }^{36}$ G. Miceli and A. Pasquarello, Phys. Rev. B 93, 165207 (2016).

${ }^{37}$ S. Birner, T. Zibold, T. Andlauer, T. Kubis, M. Sabathil, A. Trellakis and P. Vogl, IEEE Trans. Electron. Devices, 54, 2137 (2007).

${ }^{38}$ P. Li, H. Zhang, H. Li, Y. Zhang, Y. Yao, N. Palmquist, M. Iza, J. S. Speck, S. Nakamura, and S. P. DenBaars, Semiconductor Science and Technology 35, 125023 (2020).

39 J. Wang, E. C. Young, W. Y. Ho, B. Bonef, T. Margalith, and J. S. Speck, Semiconductor Science and Technology 35, 125026 (2020).

${ }^{40}$ X.Guo and E.F. Schubert, Appl. Phys. Lett. 78, 337 (2001). 


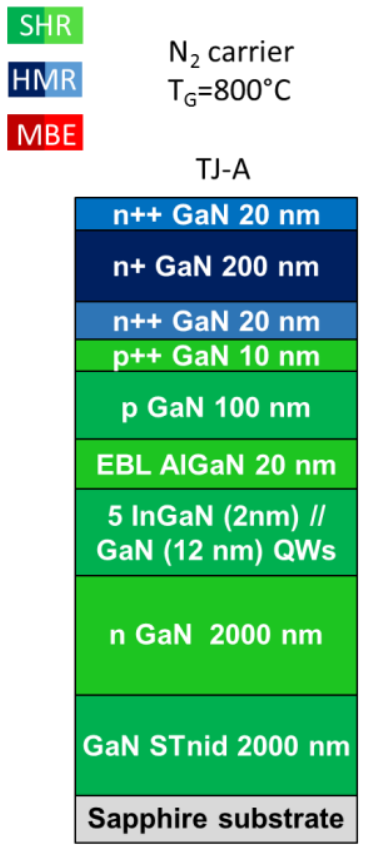

a)

\begin{tabular}{c}
$\mathrm{N}_{2}$ carrier \\
$\mathrm{T}_{\mathrm{G}}=800^{\circ} \mathrm{C}$ \\
TJ-B \\
\hline $\mathrm{n}++$ GaN $20 \mathrm{~nm}$ \\
$\mathrm{n}+\mathrm{GaN} 200 \mathrm{~nm}$ \\
\hline $\mathrm{n}++$ GaN $20 \mathrm{~nm}$ \\
\hline InGaN $4 \mathrm{~nm}$ \\
\hline $\mathrm{p}++$ GaN $10 \mathrm{~nm}$ \\
\hline $\mathrm{p}$ GaN $100 \mathrm{~nm}$ \\
\hline EBL AIGaN $20 \mathrm{~nm}$ \\
\hline 5 InGaN (2nm) // \\
GaN (12 nm) QWs \\
\hline $\mathrm{n}$ GaN $2000 \mathrm{~nm}$ \\
\hline GaN STnid $2000 \mathrm{~nm}$ \\
\hline Sapphire substrate \\
\hline
\end{tabular}

b)
$\mathrm{TJ}-\mathrm{C}$

\begin{tabular}{|c|}
\hline$n++$ GaN $20 \mathrm{~nm}$ \\
\hline $\mathrm{n}+$ GaN $200 \mathrm{~nm}$ \\
\hline $\mathrm{n}++$ GaN $20 \mathrm{~nm}$ \\
\hline $\mathrm{p}++$ GaN $10 \mathrm{~nm}$ \\
\hline $\mathrm{p}$ GaN $100 \mathrm{~nm}$ \\
\hline EBL AIGaN $20 \mathrm{~nm}$ \\
\hline $\begin{array}{l}\text { InGaN }(2 \mathrm{~nm}) / / \\
\text { GaN }(12 \mathrm{~nm}) \mathrm{QWs}\end{array}$ \\
n GaN $2000 \mathrm{~nm}$ \\
\hline GaN STnid $2000 \mathrm{~nm}$ \\
Sapphire substrate \\
\hline
\end{tabular}

c)

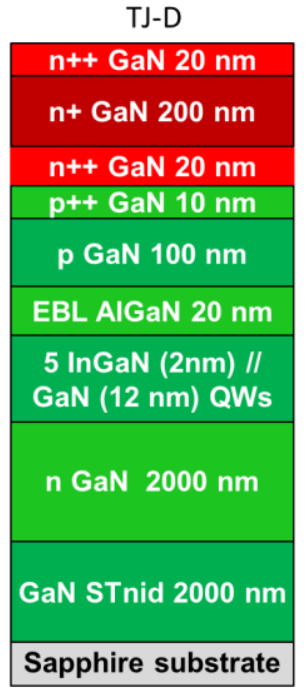

d)

Figure 1. Schematics of the different LED stacks. a) and b) are the MOCVD grown tunnel junctions (TJ$A$ and TJ-B, respectively). c) and d) are the hybrid tunnel junctions (TJ-C and TJ-D, respectively). 

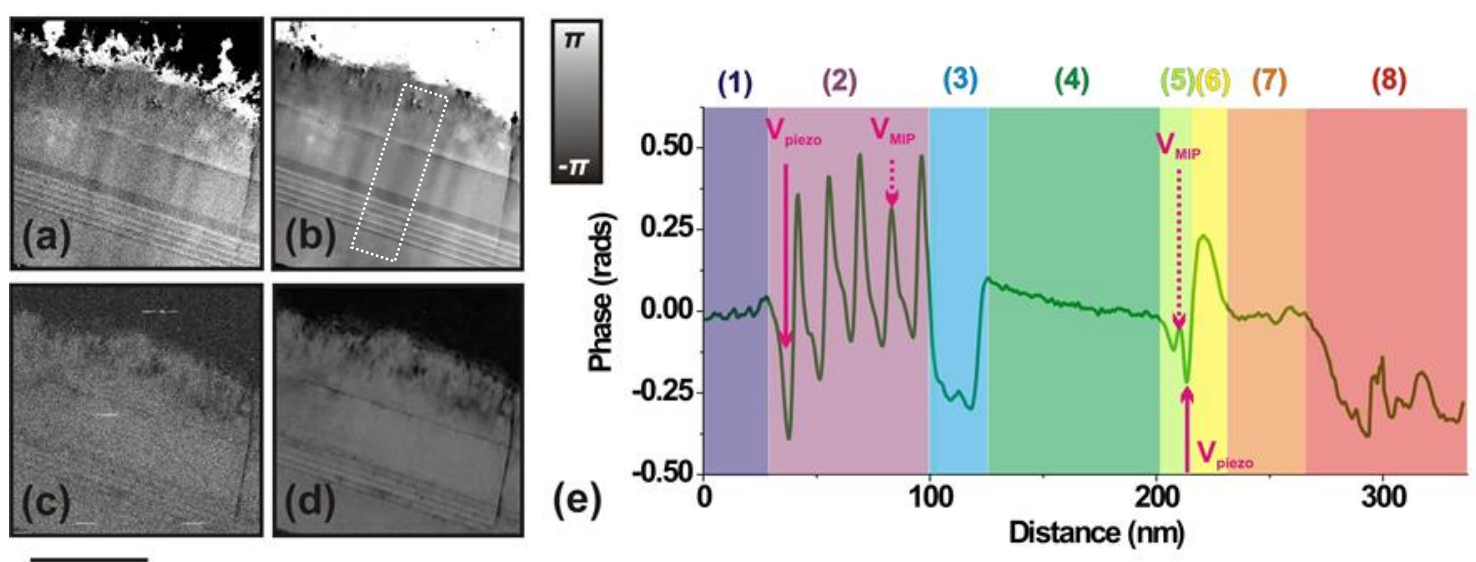

\section{$250 \mathrm{~nm}$}

Figure 2: (a) Shows phase image from TJ-B reconstructed from a single hologram acquired for $8 \mathrm{~s}$ and (b) a phase image reconstructed from a stack of 32 holograms. (c) A reconstructed amplitude image from the same single hologram and (d) from the stack of 32 holograms. (e) A phase profile extracted from the indicated region in (b). 


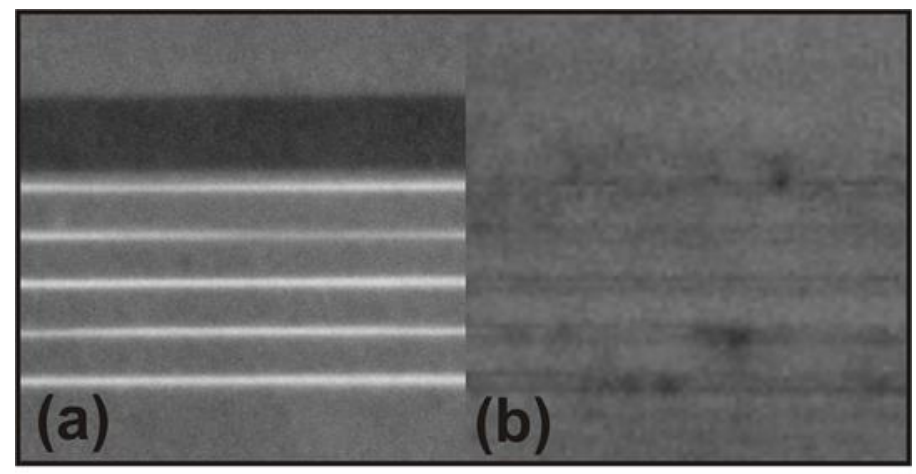

\section{$25 \mathrm{~nm}$}

Figure 3: (a) HAADF STEM image of the QWs in the TJ-B device and (b) the reconstructed amplitude image.

The dark spots in the amplitude image arise from dynamical diffraction in non-perfect InGaN regions. 

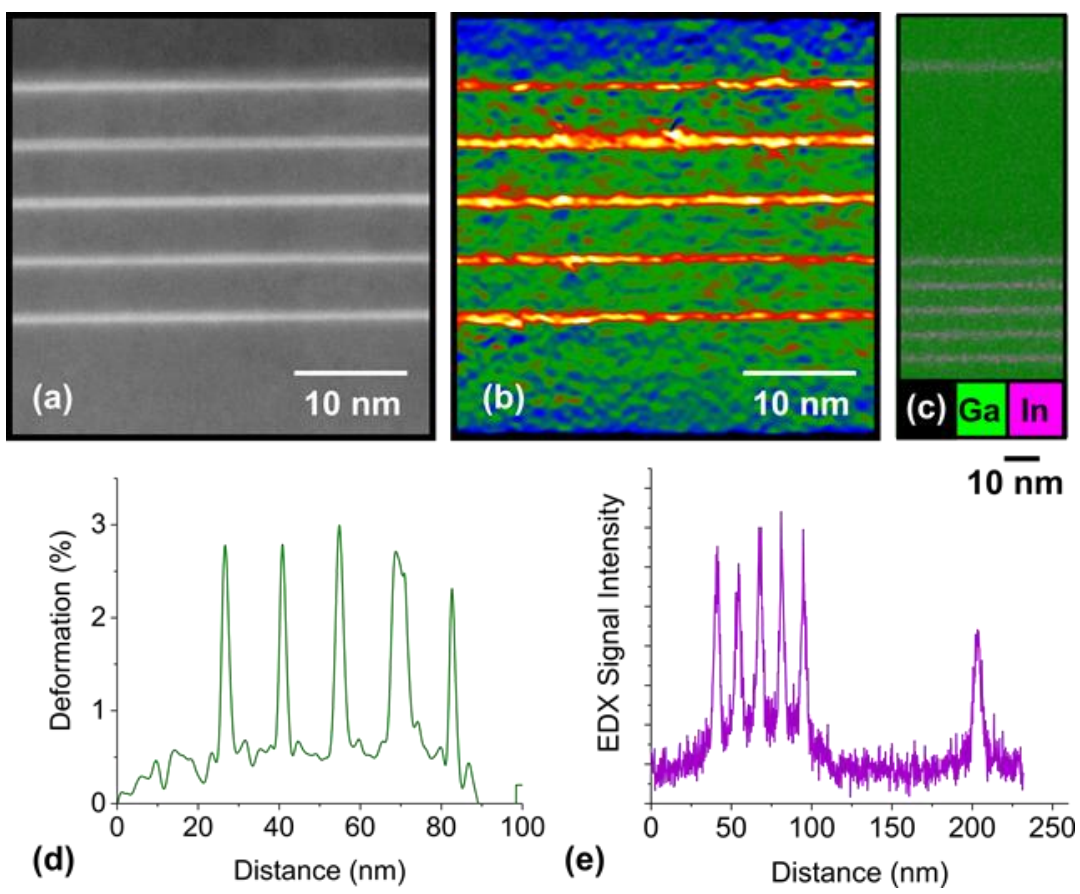

Figure 4: (a) HAADF STEM image of the QWs in the TJ-B device. (b) Deformation map for the growth direction obtained by applying a GPA algorithm to the image in (a). (c) EDX map of the TJ-B. (d) Deformation profiles taken from the region indicated in (b). (e) EDX profiles taken from the region indicated in (c). Note the EDX and GPA are not acquired from exactly the same region of the specimen. 


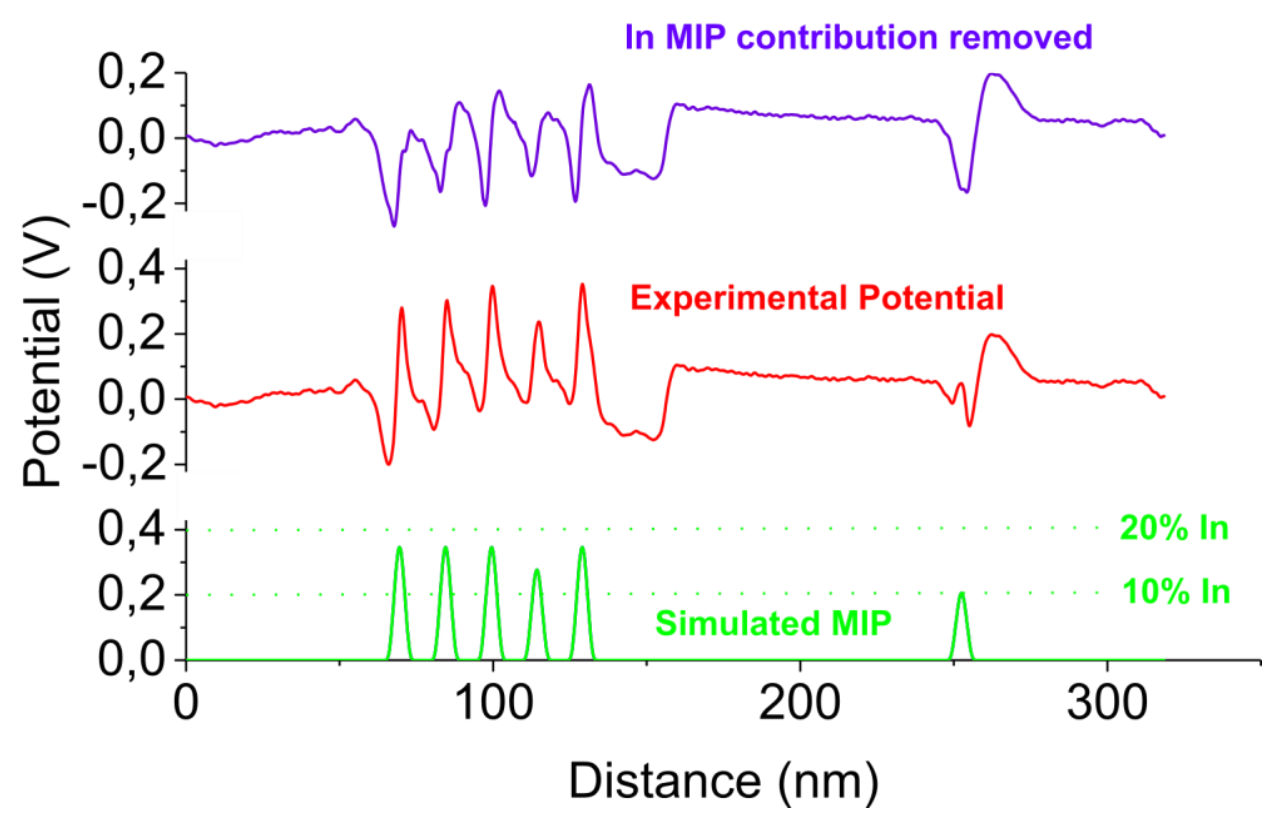

Figure 5: Simulated In MIP for the device, experimentally measured potential and the potential with the In MIP component removed. 

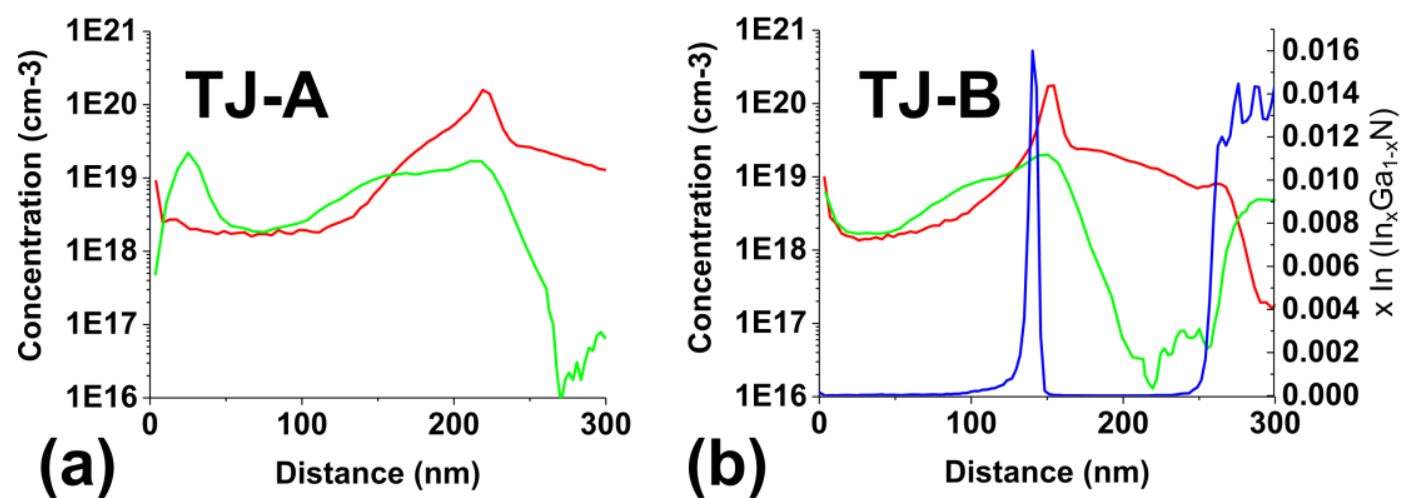

(b)

Distance (nm)
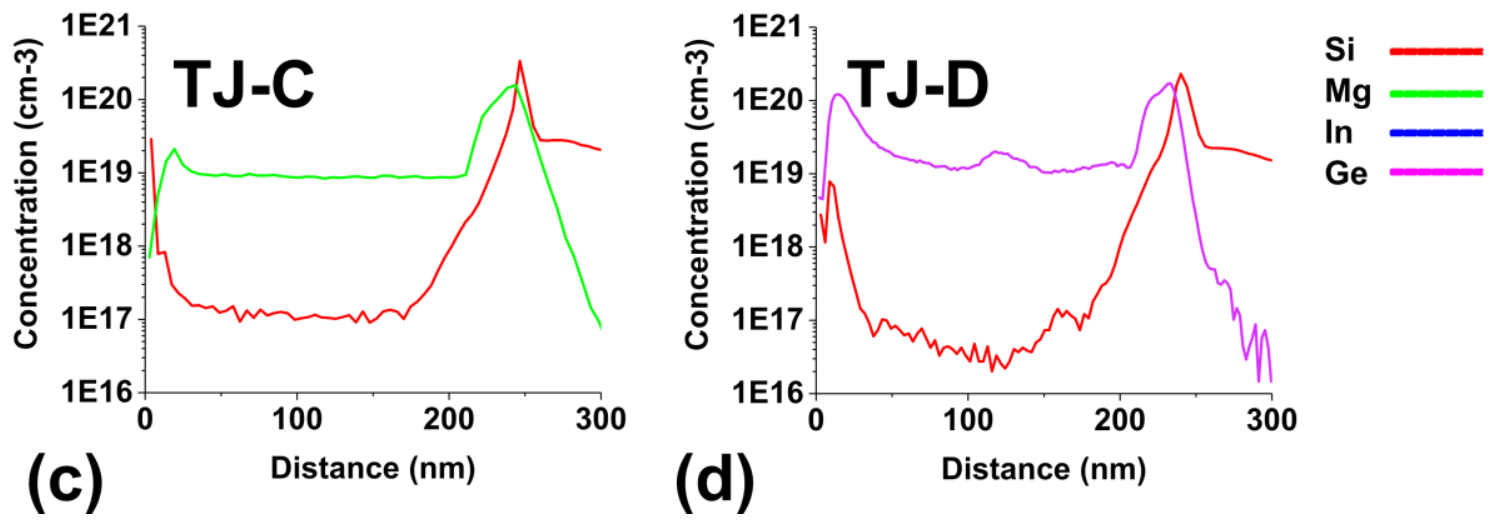

Figure 6. SIMS profiles of the MOCVD TJ without (a) and with (b) InGaN interlayer, and the hybrid TJs doped with (c) Si and (d) Ge. The top surface of the specimen is located at the left. 


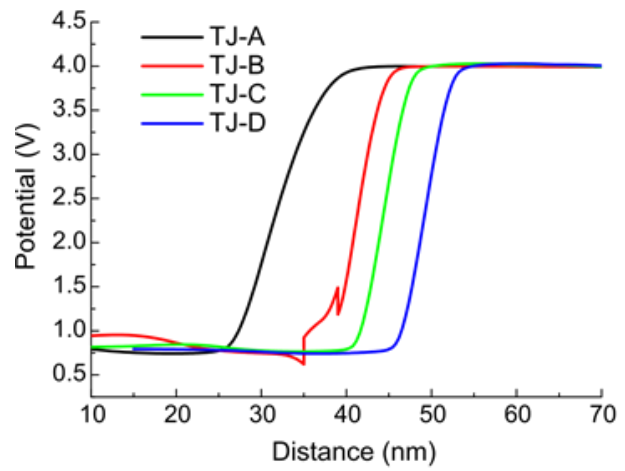

Figure 7: Simulated electric potential profiles calculated from the SIMS profiles for the TJ samples. 


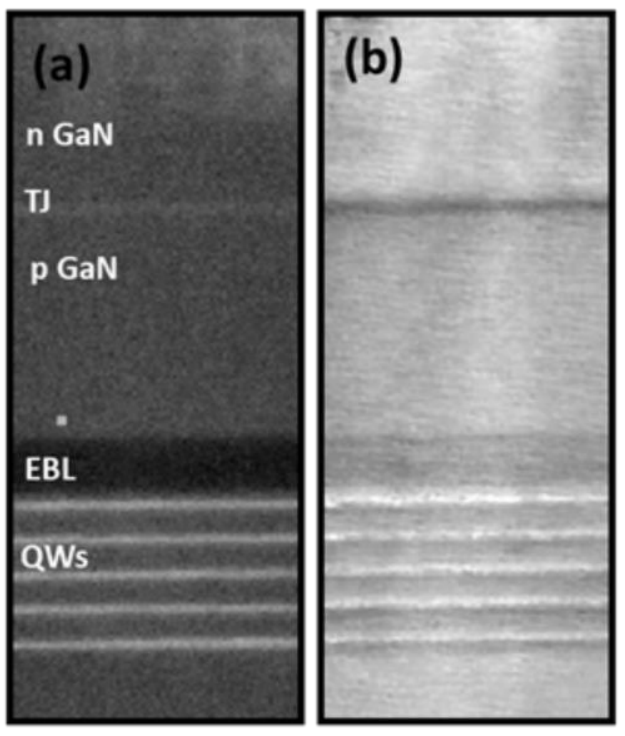

(c)
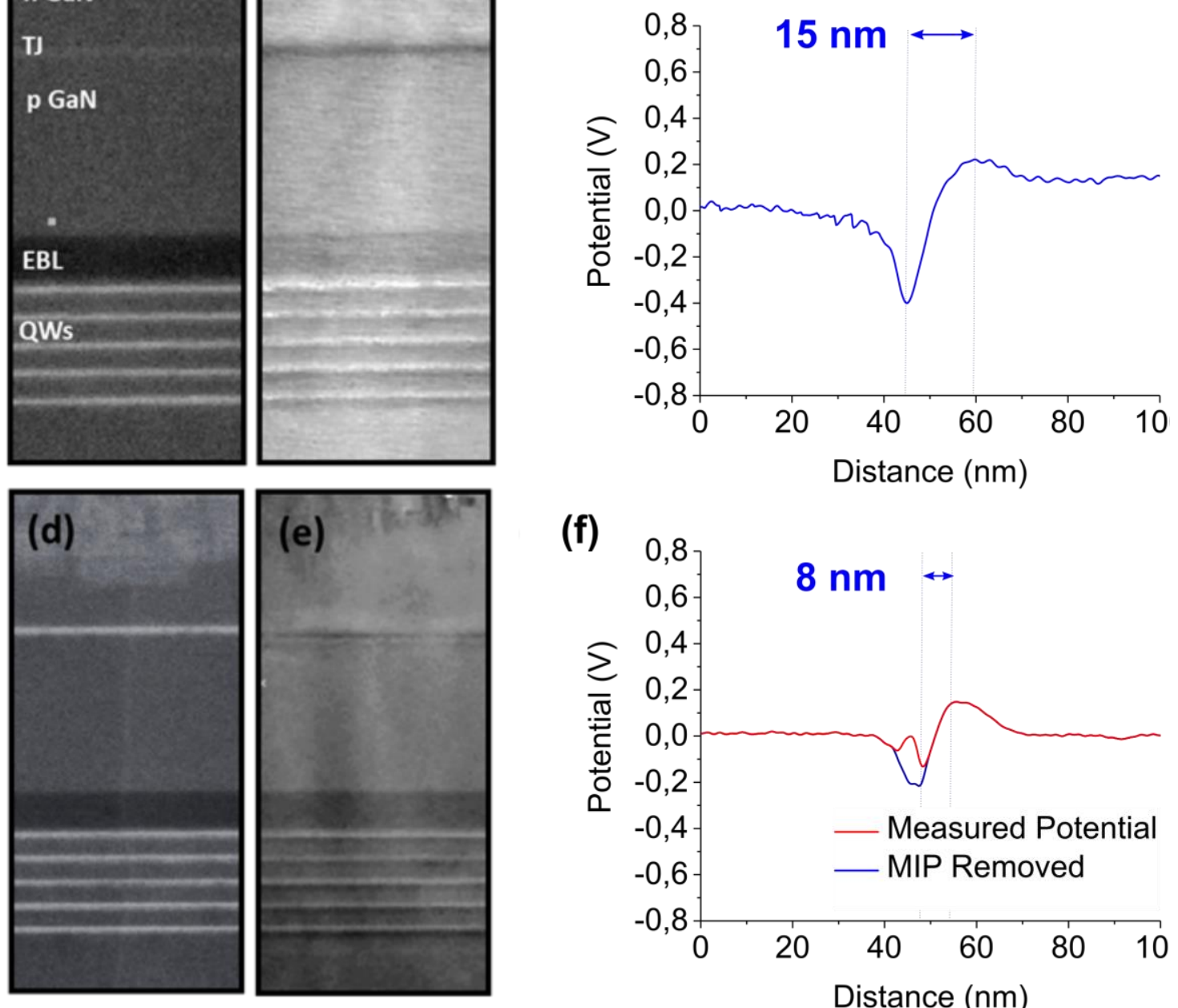

(f)
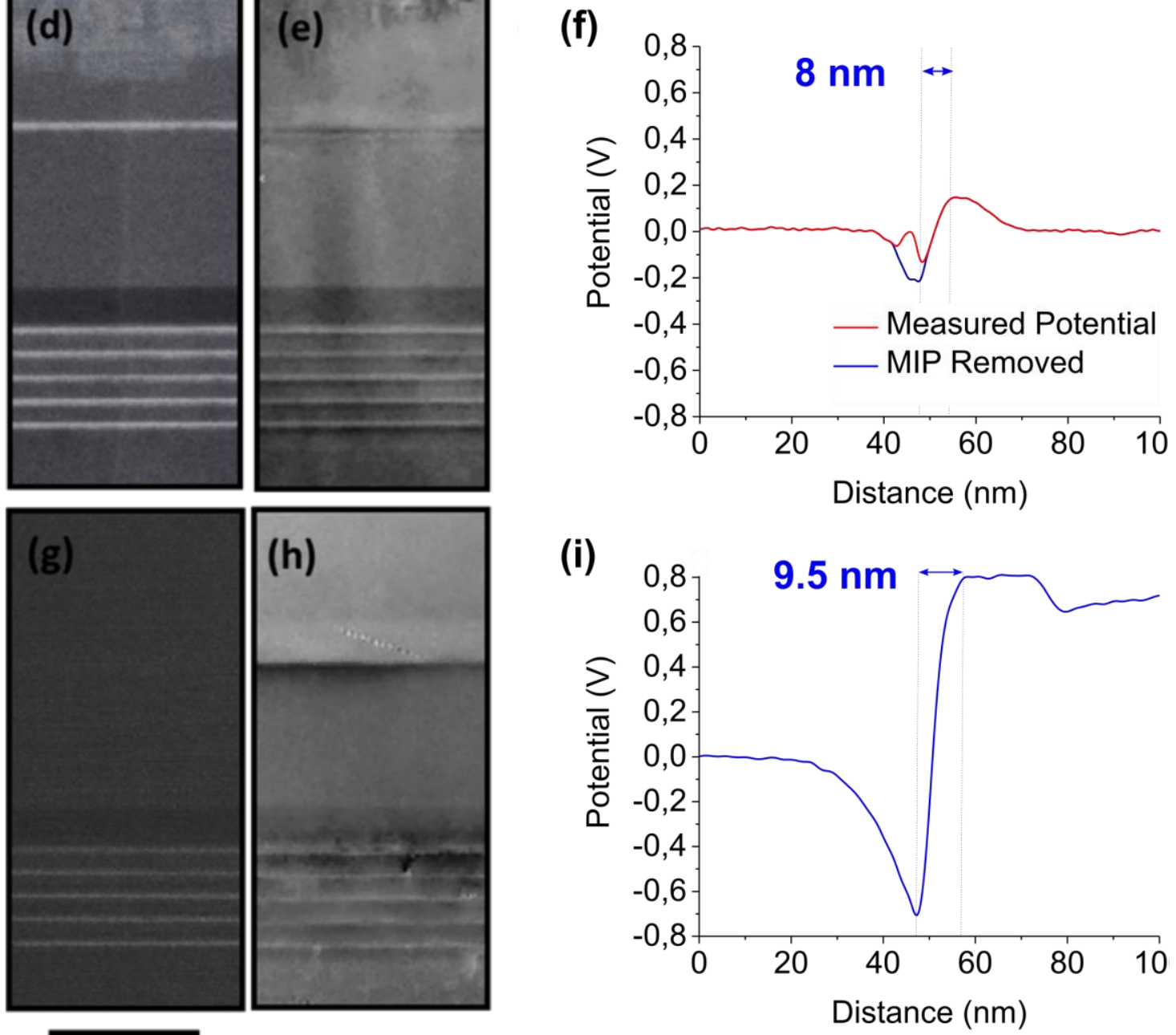

\section{$50 \mathrm{~nm}$}

Figure 8: (a) HAADF STEM images (b) potential map and (c) potential profiles for TJ-A grown at low temperature. (d) HAADF STEM image, (e) potential map and (f) potential profiles for TJ-B with interlayer. (g) HAADF STEM, (h) potential map and (i) potential profiles for TJ-C Hybrid. 

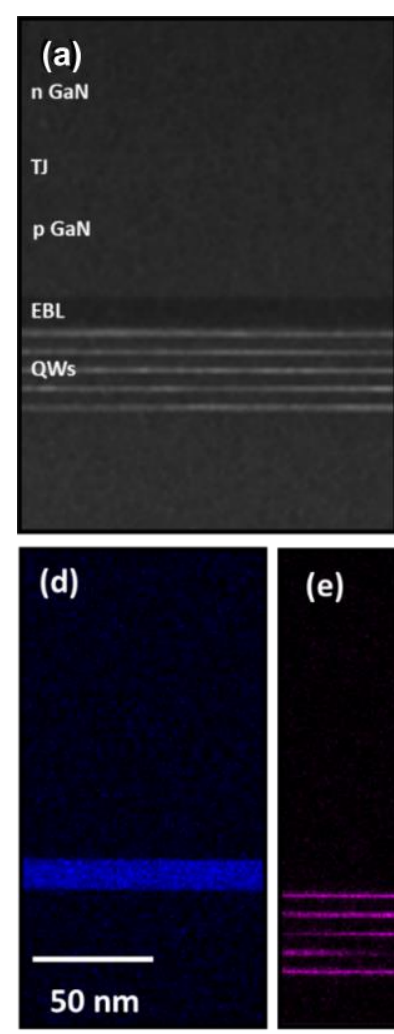

\section{(b)}

\section{(e)}

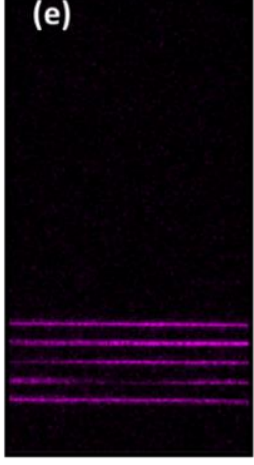

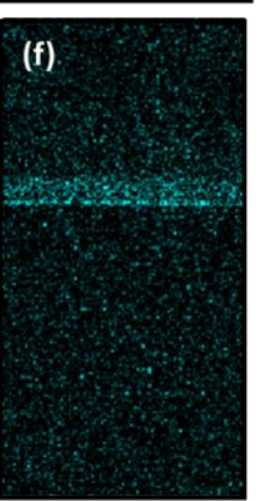

(c)
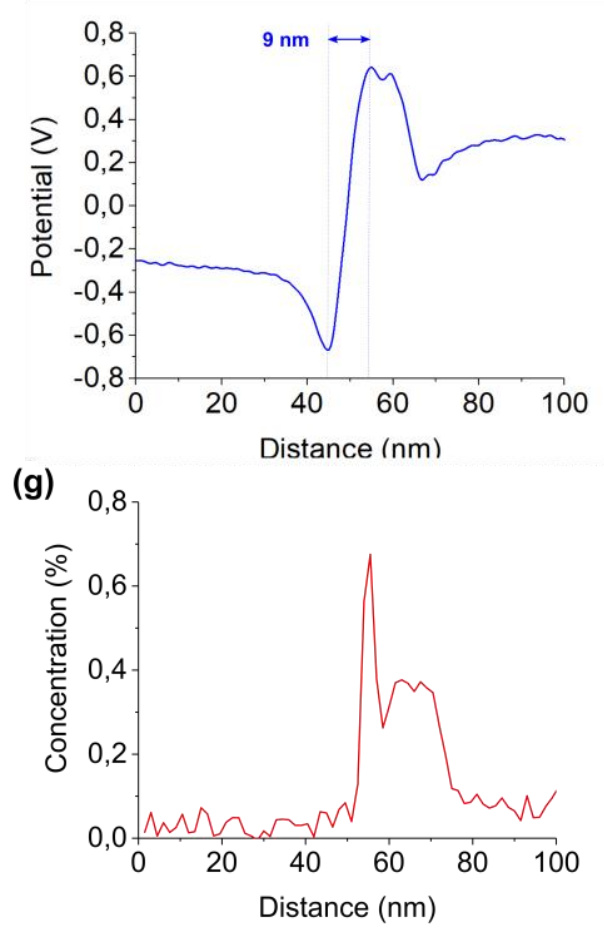

Figure 9: (a) HAADF STEM image (b) potential map and (c) potential profiles for the Ge-doped TJ-D. (d), (e) and (f) Al, In and Ge EDX maps, (g) The Ge concentration across the TJ region. 


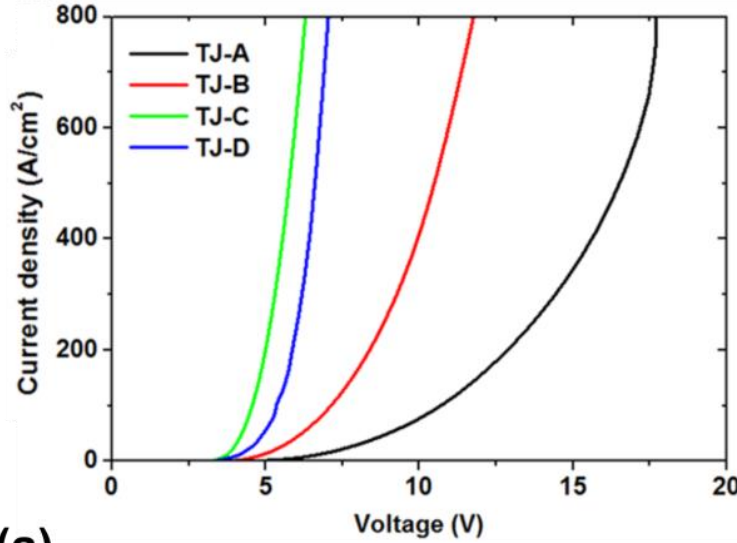

(a)

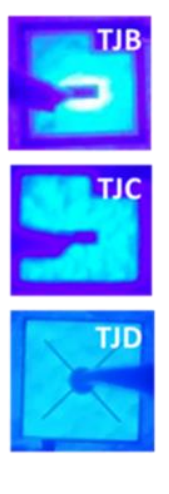

(b)

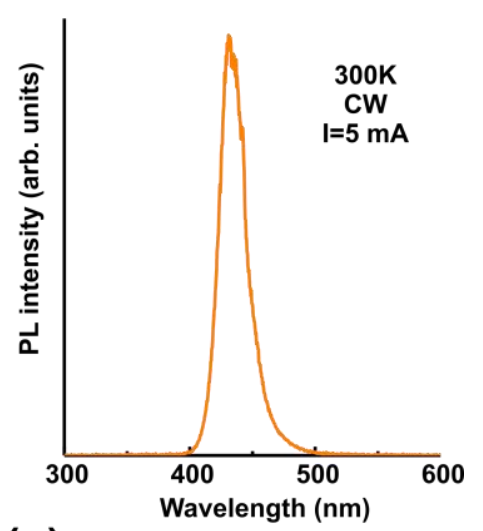

(c)

Figure 10 (a) Current density versus voltage characteristics of the tunnel junction LEDs of (100x100) $\mu \mathrm{m}^{2}$. (b) TJs $B, C$ and $D$ under operation at a current of $5 \mathrm{~mA}$. (c) Electro-luminescence spectra for TJ-D. 


\begin{tabular}{|l|l|c|c|c|c|}
\hline & Description & $\begin{array}{c}\text { TJ Width } \\
(\mathrm{nm})\end{array}$ & $\begin{array}{c}\text { Simulated TJ } \\
\text { Width } \\
(\mathrm{nm})\end{array}$ & $\begin{array}{c}\text { Specimen } \\
\text { thickness } \\
(\mathrm{nm})\end{array}$ & $\begin{array}{c}\text { Measured step } \\
\text { in potential } \\
(\mathrm{V})\end{array}$ \\
\hline TJ-A & Low Temperature & $15+/-2$ & 15 & $440+/-5$ & $0.62+/-0.05$ \\
\hline TJ-B & $\begin{array}{l}\text { Low Temperature } \\
\text { Interlayer }\end{array}$ & $8+/-2$ & 10 & $240+/-5$ & $0.36+/-0.05$ \\
\hline TJ-C & Hybrid Si doped & $9.5+/-2$ & 8 & $325+/-5$ & $1.50+/-0.05$ \\
\hline TJ-D & Hybrid Ge doped & $9+/-2$ & 7 & $315+/-5$ & $1.29+/-0.05$ \\
\hline
\end{tabular}

Table 1: Measured properties for the different TJ specimens. The thickness of the examined TEM lamellas are shown. 